\title{
Rotura de la vena dorsal superficial de pene
}

\author{
J. Arce Gil, J.M. Rodríguez-Ledesma, H. Villavicencio Mavrich \\ Servicio de Urología. Fundació Puigvert. Barcelona.
}

Actas Urol Esp 2006; 30 (2): 215-217

\section{RESUMEN \\ ROTURA DE LA VENA DORSAL SUPERFICIAL DE PENE}

La rotura de vena dorsal superficial de pene durante el coito es una entidad poco frecuente que plantea el diagnóstico diferencial con otras lesiones peneanas agudas que pueden llegar a requerir exploración quirúrgica. Presentamos el caso de un paciente varón de 58 años con hematoma peneano no doloroso tras el coito, de 24 horas de evolución con una evolución y exploración física características que permitió adoptar una actitud conservadora con recuperación total sin secuelas

Palabras clave: Vena dorsal superficial del pene. Lesiones vasculares peneanas. Lesiones coitales. Hematoma peneano

\section{ABSTRACT \\ RUPTURE OF THE SUPERFICIAL DORSAL VEIN OF THE PENIS}

Rupture of the superficial dorsal vein of the penis during intercourse is an unfrequented entity that makes the differential diagnosis with other acute penile injuries that may require surgical exploration necessary. We report the case of a 58-year-old male patient with 24-hours evolution painless haematoma after intercourse; its evolution and characteristic physical exploration enable us to adopt a conservative approach that resulted in complete recovery without sequels

Keywords: Superficial dorsal vein of the penis. Penile vascular lesions. Coital injuries. Penile haematoma.

$\mathrm{E}$ estado de erección los tegumentos y estructuras vasculares del pene pierden su elasticidad disminuyendo su resistencia frente a los traumatismos. Dentro de las estructuras vasculares del pene se incluye la vena dorsal superficial del pene y su ingurgitación durante la erección la hace susceptible de rotura.

Aunque la incidencia real de rotura de vena superficial de pene no es conocida existen contadas publicaciones de casos clínicos aislados. La evolución y los hallazgos físicos en esta entidad son característicos, permitiendo una aproximación diagnóstica certera dentro del diagnóstico diferencial del traumatismo peneano agudo.

\section{CASO CLÍNICO}

Paciente varón de 58 años de edad con antecedente de postectomía como único antecedente médico-quirúrgico conocido que acude al servicio de urgencias por presentar hematoma no doloroso en cara dorsal del pene de 24 horas de evolución tras relación sexual vigorosa el paciente no refiere antecedente de chasquido ni detumescencia peneana durante el coito. No presenta hematuria ni sintomatología miccional.

A la exploración física se observa un hematoma en toda la extensión del pene con predominio de la cara dorsal desde la raíz peneana hasta la porción distal respetando el glande, no doloroso a la palpación. No se objetiva deformidad-incurvación peneana y no se palpan defectos de continuidad.

Por su presentación clínica y sus hallazgos físicos se orienta el cuadro como una rotura de vena superficial del pene por lo que se decide una actitud terapéutica conservadora, administración de hielo local, heparinoides tópicos y abstinencia sexual hasta control clínico posterior (Figs. 1 y 2). 


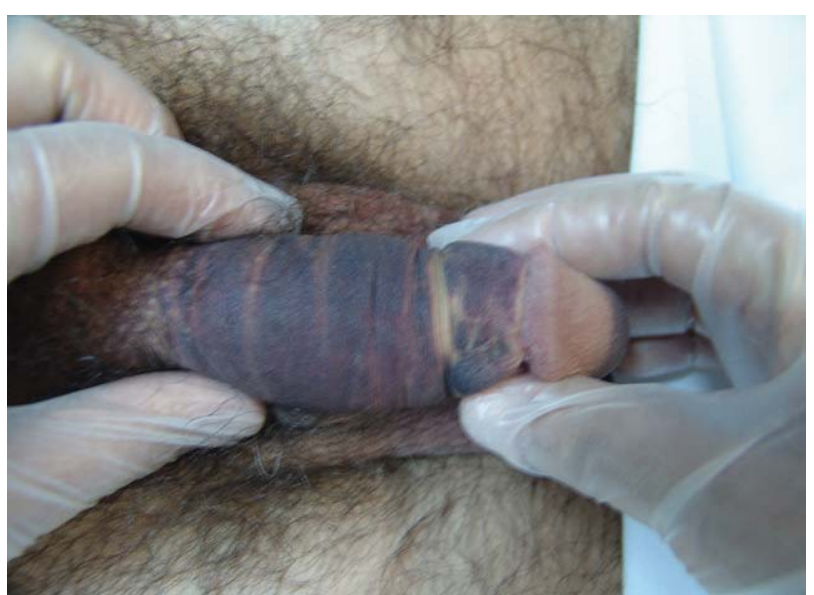

FIGURA 1

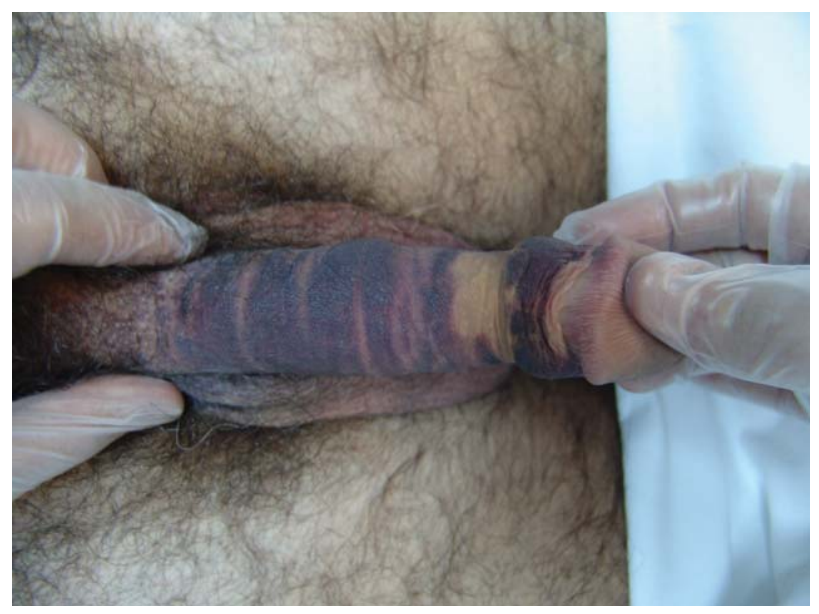

\section{FIGURA 2}

Se realizó control clínico evolutivo en las 2 semanas posteriores al cuadro objetivando desaparición completa del hematoma peneano, sin deformidades ni lesiones cutáneas, permaneciendo asintomático en todo momento. Posteriormente las relaciones sexuales fueron satisfactorias sin repercusión ni clínica asociada en las erecciones.

\section{DISCUSIÓN}

Múltiples venas superficiales discurren sobre la superficie dorsolateral del pene situadas entre las fascias de Colles y de Buck. Estas venas se unen a nivel del hilio peneano para formar la vena dorsal superficial del pene, la cual puede ser única o doble. Esta vena drena la sangre venosa procedente del tejido situado por encima de la fascia de Buck, que incluye la piel y el dartos del pene, desembocando en la vena safena izquierda en la mayoría de los casos.
Las lesiones vasculares peneanas adquiridas pueden ser clasificadas en simples y complejas en función de si la lesión vascular es única (arterias o venas dorsales, cuerpos cavernosos, arteria frenular, linfáticos) o va asociada a lesión de otras estructuras (uretra, fascia de Buck, ligamentos, lesión testicular) ${ }^{1}$.

Durante el acto sexual las arterias y venas de la superficie dorsal del pene se interponen entre los cuerpos cavernosos y las restantes estructuras óseas circundantes de la vagina siendo susceptibles de rotura debido a su incrementada vascularidad y disminución de la resistencia durante la erección.

La mayoría de las lesiones vasculares coitales adquiridas suelen ocurrir con la flexión ventral o dorsal forzada del pene erecto durante el coito pero también puede acontecer cuando el pene accidentalmente se desliza fuera de la vagina durante el coito chocando contra la sínfisis del pubis o el periné. Infrecuentemente el pene erecto puede ser lesionado al girarse o caerse de la cama, durante la masturbación o por lesión directa sobre el pene. Otros factores que pueden producir lesiones vasculares son las mordeduras y las lesiones auto-inflingidas ${ }^{1}$.

Los signos y síntomas clínicos de las lesiones vasculares agudas del pene consisten en aparición súbita de dolor, presencia de chasquido, edema, detumescencia, equimosis y/o hematoma, sangrado uretral, defecto de continuidad palpable sobre la lesión o desviación del pene contralateral al lado afecto.

El mecanismo de lesión en lesiones de la vena dorsal superficial del pene durante el coito es básicamente el mismo que la ruptura de la vena dorsal profunda y la del cuerpo cavernoso.

En la rotura de los cuerpos cavernosos el cuadro típico es el antecedente de un chasquido seguido de dolor súbito y detumescencia inmediata, edema, equímosis y deformidad del pene con incurvación hacia el lado opuesto al del cuerpo cavernoso afecto. El mantenimiento de la erección es un signo claro de la indemnidad de los cuerpos cavernosos. Si existiera compromiso uretral puede referir sindrome uretral, uretrorragia y/o hematuria ${ }^{2,3}$. La localización del hematoma dependerá de la indemnidad de las diferentes fascias. 
La presentación de la ruptura de la vena dorsal profunda del pene es esencialmente indistinguible de la ruptura de cuerpos cavernosos debiéndose considerar dentro del diagnóstico diferencial del pene agudo $^{3-5} \mathrm{si}$ bien se puede presentar sin dolor ni chasquido peneano ${ }^{5}$.

En contraste, en la rotura de la vena dorsal superficial del pene, el paciente refiere una detumescencia gradual y no súbita con ausencia de chasquido y hematoma progresivo, en la mayoría de casos en forma de semiluna. Así, la extravasación de sangre progresará a través del tejido subcutáneo del pene con indemnidad de la fascia de Buck y de Colles. El paciente no suele presentar dolor y la exploración, la cual no es dolorosa, no demostrará deformidades ni defectos de continuidad ${ }^{6,7}$.

El trauma repetido sobre la vena dorsal superficial del pene puede presentarse como erecciones dolorosas, equimosis, apreciándose un cordón palpable causado por la trombosis de la vena dorsal superficial del pene, denominándose enfermedad de Mondor ${ }^{1,8-11}$.

Es importante una buena anamnesis y una buena exploración física ya que el diagnóstico de rotura de vena dorsal superficial del pene en la mayoría de los casos es fundamentalmente clínico. En caso de duda puede ser de utilidad la realización de una prueba barata de fácil acceso y eficaz como la ecografia peneana, aportándonos información sobre la integridad de la túnica albugínea $^{3}$. En caso de duda diagnóstica y dependiendo de la severidad se podría requerir la exploración quirúrgica inmediata ${ }^{2}$.

A pesar de los pocos casos descritos hasta la fecha, el tratamiento de elección en la rotura de la vena dorsal superficial del pene es conservador administración de antinflamatorios, hielo local, cremas heparinoides y vendaje compresivo dependiendo de la severidad, indicándose reposo sexual durante al menos 2 semanas sin apreciar- se secuelas posteriores, conservando la capacidad eréctil previa y sin lesiones cutáneas ni incurvaciones peneanas.

\section{REFERENCIAS}

1. Lee J, Singh B, Kravets FG, Trocchia A, Waltzer WC, Khan SA. Sexually acquired injuries of the penis: A review. J Trauma 2000 Aug;49(2):351-358.

2. Marino del Real J, Senovilla Pérez JL, Mateos Blanco J, Abengózar García-Moreno A, Sevilla Zabaleta M, Caballero Gómez M, Cabello Radial J, Murillo Mirat J. Fractura de pene. Revisión de la literatura y presentación de dos casos. Actas Urol Esp. 2000;24(9):767-770.

3. Vera Donoso CD, Vidal J, García Reboll L, Boronat Tormo F, Ruiz Cerdá JL, Jiménez-Cruz JF. Diagnóstico y tratamiento del traumatismo peneano coital. Actas Urol Esp 1991;15(4):397-400.

4. Nicely ER, Costaible RA, Moul JW. Rupture of the deep dorsal vein of the penis during sexual intercourse $J$ Urol 1992 Jan; 147(1):150-152.

5. Herreo Polo E, Megias Garrigos J, Puertas Ruiz M, Torrus Tendero P, Sánchez Marcos M. Hematoma peneano por rotura de vena dorsal profunda durante el coito. Arch Esp Urol 2000 Jun;53(5):473-475.

6. Waller DA, Britton JP, Ferro MA. Rotational injury of the penis. Br J Urol 1990 65(4):425.

7. Bujons Tur A, Rodríguez-Lesdesma JM, Cetina Errando A, Puigvert Martínez A, Iglesias Guzmán JC, Villavicencio Mavrich H. Hematoma peneano secundario a rotura de la vena dorsal superficial del pene. Arch Esp Urol 2004;57(7): 748-751.

8. Bird V, Krasnokutsky S, Zhou HS, Jarahy R, Khan SA. Traumatic thrombophlebitis of the superficial dorsal vein of the penis: an occupational hazard. Am J Emerg Med 1997; 15(1):67-69.

9. Nehru-Babu M, Hendry D, Ai-Saffar N. Rupture of the dorsal vein mimicking fracture of the penis. BJU Int 1999; 84(1):179-180.

10. Ganem JP, Kennelly MJ. Ruptured Mondor's disease of the penis mimicking penile fracture. J Urol 1998;159(4):1302.

11. Herreo Payo A, Páez Borda A, Martín Oses E, Luján Galán M, Berenguer Sánchez A. Una causa inusual de deformidad peneana aguda: La trombosis de la vena superficial del pene. Arch Esp Urol. 1996 Oct;49(8):861-864.

Dr. J. Arce Gil

E-mail: Jacobo1976@hotmail.com

(Trabajo recibido el27 de mayo de 2005) 